\title{
Guest Editorial: search-based software engineering
}

\author{
Gordon Fraser • Jerffeson Teixeira de Souza
}

Published online: 4 May 2014

(C) Springer Science+Business Media New York 2014

With modern computer systems growing in size and complexity, the demands to functionality, scalability, and robustness of software engineering techniques present ever new challenges. Search-based software engineering (SBSE) has emerged as a promising direction of research that can successfully counter some of these challenges.

SBSE involves the automatic - or semi-automatic - resolution of complex software engineering problems modeled as optimization problems, using search algorithms, in particular metaheuristics. An exponential growth of the number of published papers in this area in the last decade bears witness to the popularity search-based approaches within the software engineering research community, and SBSE has been successfully applied to solve problems in nearly all software development life cycle phases.

This issue presents three articles that are all based on search-techniques, yet tackle very different aspects of software engineering:

The paper "An Experimental Evaluation of the Importance of Randomness in Hill Climbing Searches applied to Software Engineering Problems" by Marcio Barros addresses a fundamental, cross cutting concern related to the importance of randomness in SBSE. A range of experiments analyzes whether quasi-random sequences could be employed as an alternative to classic pseudo-random number generators. Contrary to past experiments in this domain the author finds no evidence that quasi-random sequences would outperform pseudorandom sequences.

Eric Fredericks, Byron DeVries, and Betty Cheng, in their paper "AutoRELAX: Automatically RELAXing a Goal Model to Address Uncertainty", address the problem of specifying requirements for dynamic adaptive systems such as for example robotic systems, where there is uncertainty about the environment during requirements elicitation. The article proposes and evaluates an approach based on search-techniques to generate goal models based on such uncertain environmental conditions.

Finally, the paper "Evolving Robust Networks for Systems-of-Systems: is it Viable for Large Networks?" by Jonathan Aitken, Rob Alexander, Tim Kelly, and Simon Poulding looks at software systems using ad-hoc networks, and applies search techniques to optimize these

\footnotetext{
G. Fraser $(\bowtie)$

Department of Computer Science, University of Sheffield, Sheffield S1 4DP, UK e-mail: gordon.fraser@sheffield.ac.uk

J. T. de Souza

Optimization in Software Engineering Group, State University of Ceará, Fortaleza 60740-903, Brazil

e-mail: jerffeson.souza@uece.br
} 
networks. Initial empirical evidence is encouraging, and the tool implementing the technique can produce solutions that are better than a human engineer could come up with.

These three exciting articles demonstrate the versatility of SBSE, and witness how broad and dynamic the SBSE community is. We hope you enjoy reading them as much as we did.

We wish to thank all authors and reviewers for their time and effort, and Victor Basili and Lionel Briand, the Editors-in-Chief of the Empirical Software Engineering journal and the editorial team for their support.

\section{Guest editors}

Gordon Fraser and Jerffeson Teixeira de Souza

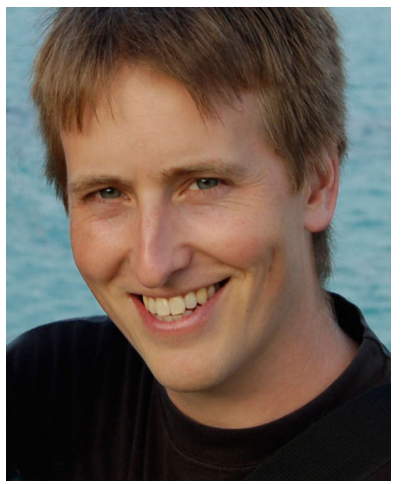

Gordon Fraser is a lecturer in Computer Science at the University of Sheffield, UK. He received a $\mathrm{PhD}$ in computer science from Graz University of Technology, Austria, in 2007. The central theme of his research is improving software quality, and his recent research concerns the prevention, detection, and removal of defects in software. More specifically, he develops techniques to generate test cases automatically, and to guide the tester in validating the output of tests by producing test oracles and specifications.

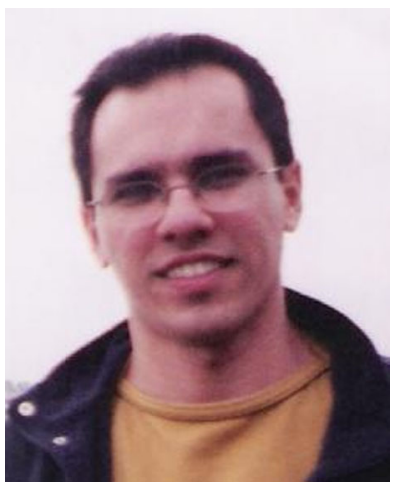

Jerffeson Teixeira de Souza is a full professor and the dean of research and graduate studies at State University of Ceará (UECE), Brazil. He holds a Ph.D. in Computer Science from the School of Information Technology and Engineering (SITE) of University of Ottawa, Canada. His main research interests are: search-based software engineering, multi-objective evolutionary algorithms, machine learning and software patterns. 